\title{
ON THE NONSINGULARITY OF COMPLEX MATRICES
}

\author{
PaUl Camion* and A. J. HofFman**
}

\begin{abstract}
Let $A=\left(a_{i j}\right)$ be a real square matrix of order $n$ with nonnegative entries, and let $M(A)$ be the class of all complex matrices $B=\left(b_{i j}\right)$ of order $n$ such that, for all $i, j,\left|b_{i j}\right|=a_{i j}$. If every matrix in $M(A)$ is nonsingular, we say $M(A)$ is regular, and it is the purpose of this note to investigate conditions under which $M(A)$ is regular.
\end{abstract}

Many sufficient conditions have been discovered (cf., for instance, [8] and [3], and their bibliographies), motivated by the fact that the negation of these conditions, applied to the matrix $B-\lambda I$, yields information about the location of the characteristic roots. We shall show that a mild generalization of the most famous conditions [2] is not only sufficient but also necessary. (The application of our result to characteristic roots will not be discussed here, but is contained in [5]. See also [7] and [9]). If

$$
a_{i i}>\sum_{i \neq j} a_{i j}, \quad i=1, \cdots, n,
$$

then ([2]) $M(A)$ is regular. Clearly if $P$ is a permutation matrix, and $D$ a diagonal matrix with positive diagonal entries, such that $P A D$ satisfies (1.1), then $M(A)$ is regular. We shall show that, conversely, if $M(A)$ is regular, there exist such matrices $P$ and $D$ so that (1.1) holds.

2. Notation and lemmas, If $x=\left(x_{1}, \cdots, x_{n}\right)$ is a vector, $x^{D}$ is the diagonal matrix whose $i$ th diagonal entry is $x_{i}$. If $M=\left(m_{i j}\right)$ is a matrix, $M^{v}$ is the vector whose $i$ th coordinate is $m_{i i}$. A vector $x=$ $\left(x_{1}, \cdots, x_{n}\right)$ is positive if each $x_{j}>0 ; x$ is semi-positive if $x \neq 0$ and each $x_{j} \geqq 0$. A diagonal matrix $D$ is positive (semipositive) if $D^{v}$ is positive (semi-positive). If $A=\left(a_{i j}\right)$ is a matrix with nonnegative entries, a particular entry $a_{i j}$ is said to be dominant in its column if

$$
a_{i j}>\sum_{k \neq i} a_{k j}
$$

LEMMA 1. If $e_{1}, \cdots, e_{n}$ are nonnegative numbers such that the largest does not exceed the sum of the others, then there exist complex numbers $z_{i}$ such that

Received June 29, 1964. *Euratom

** The work of this author was supported in part by the Office of Naval Research under Contract No. Nonr 3775(00), NR 047040. 


$$
\left|z_{i}\right|=e_{i}
$$$$
i=1, \cdots, n
$$

and

$$
\Sigma z_{i}=0
$$

Proof. It is geometrically obvious (and can easily be proved by induction) that the conditions on $\left\{e_{i}\right\}$ imply there exists a (possibly degenerate) polygon in the complex plane whose successive sides have length $e_{1}, e_{2}, \cdots, e_{n}$. Let the vertices $x_{1}, \cdots, x_{n}$ be so numbered that $\left|x_{i}-x_{i+1}\right|=e_{i}, i=1, \cdots, n-1,\left|x_{n}-x_{1}\right|=e_{n}$. Setting $z_{i}=x_{i}-x_{i+1}$ obviously satisfies (2.1) and (2.2).

Lemma 2. Let $M$ be a real matrix with $m$ rows and $n$ columns. Then

$$
M x \leqq 0, \quad x \text { semi-positive }
$$

is inconsistent if and only if

$$
w^{\prime} M>0, \quad w \geqq 0
$$

is consistent. Further, if (2.4) holds, we may assume there exists $a w$ satisfying (2.4) with at most $n$ coordinates of $w$ positive.

This lemma is well known in the theory of linear inequalities.

3. THEOREM. Let $A=\left(a_{i j}\right)$ be a matrix of order $n$ with each entry nonnegative. The following statements are equivalent:

(3.1) $M(A)$ is regular:

(3.2) if $D$ is any semi-positive diagonal matrix, then $D A$ contains an entry dominant in its column;

(3.3) there exists a permutation matrix $P$ and a positive diagonal matrix $D$ such that $P A D$ satisfies (1.1).

Proof. (3.1) $\Longrightarrow(3.2)$. Assume (3.2) false for some semipositive $D$ with $D^{v}=\left(d_{1}, \cdots, d_{n}\right)$. Let $\left(a_{1 j} d_{1}, \cdots, a_{n j} d_{n}\right)$ be any column vector of $D A$. The coordinates of this vector satisfy the hypotheses of Lemma 1 , so there exist complex numbers $z_{1}, \cdots, z_{n}$ satisfying

$$
\Sigma z_{i}=0
$$

and

$$
\left|z_{i}\right|=a_{i j} d_{i}, \quad i=1, \cdots, n
$$


Let

$$
b_{i j}=a_{i j} z_{i}|| z_{i} \mid,
$$

with $z_{i} /\left|z_{i}\right|=1$, if $z_{i}=0$. Then (3.4) and (3.5) become

$$
\sum d_{i} b_{i j}=0 \text {, }
$$

and

$$
\left|b_{i j}\right|=a_{i j}, \quad i, j=1, \cdots, n .
$$

But (3.7) states $B \in M(A)$, and (3.6) - since not all $d_{i}$ are 0 -asserts a linear dependence among the rows of $B$. Thus $B \in M(A)$ would be singular, violating (3.1).

$(3.2) \Rightarrow(3.3)$. Let $K$ be a matrix of order $n$ with $k_{i i}=1, k_{i j}=$ -1 for $i \neq j$, and let $A_{j}$ be the $j$ th column of $A$. Consider the system of $n^{2}$ linear inequalities in the semi-positive vector $x$

$$
K A_{j}^{D} x \leqq 0, \quad j=1, \cdots, n .
$$

Notice that (3.2) is identical with the statement that (3.7) is inconsistent. By Lemma 2, there exist $n$ nonnegative vectors $\mu^{1}, \cdots, \mu^{n}$ such

$$
\sum_{j} \mu^{j^{\prime}} K A_{j}^{D}>0
$$

Let $\mu^{j}=\left(\mu_{1}^{j}, \cdots, \mu_{n}^{j}\right)$. By the last sentence of Lemma 2, we may assume at most $n$ of the $n^{2}$ numbers $\left\{\mu_{k}^{j}\right\}$ are positive.

Since each row of each $K A_{j}^{D}$ contains at most one positive entry, it follows from (3.8) that exactly $n$ of the $\left\{\mu_{k}^{j}\right\}$ are positive. We now show that, for each $j$, there is exactly one $k$ such that $\mu_{k}^{j}>0$. Assume otherwise, then for (say) $j=j^{*}, \mu^{j^{*}}=0$. Let $\tilde{A}$ be the matrix obtained from $A$ by replacing $A_{j *}$ by 0 . Then (3.8) would still hold with $A_{j}$ replaced by 0 , so (from the "only if" part of Lemma 2), for any semipositive diagonal matrix $E, E \widetilde{A}$ contains an entry dominant in its column. Let $y$ be a real nonzero vector orthogonal to the columns of $\widetilde{A}$, let $N=\left\{i \mid y_{i} \geqq 0\right\}$, and $N^{\prime}$ the complementary set of indices. Then, for each $j$.

$$
\sum_{i \in N} y_{i} a_{i j}=\sum_{i \in N^{\prime}}\left(-y_{i}\right) a_{i j}
$$

If $E$ is the diagonal matrix with $E^{r}=\left(\left|y_{1}\right|, \cdots,\left|y_{n}\right|\right)$, then $E \widetilde{A}$, from (3.9), would contain no entry dominant in its column, a contradiction.

Let $\sigma$ be the mapping sending $j \rightarrow k$, where $\mu_{k}^{j}>0$. By (3.8), $\sigma$ is a permutation of $\{1, \cdots, n\}$, and

$$
a_{i, \sigma^{-1} i} \mu_{i}^{\sigma^{-1} i}>\sum_{j \neq i} a_{i, \sigma^{-1} j} \mu_{j}^{\sigma^{-1} j}, \quad i=1, \cdots, n,
$$

which is $(3.3)$. 
$(3.3) \Longrightarrow(3.1)$ was noted in the introduction.

4. Remarks. (i) It is perhaps worth pointing out that the permutation in (3.3) is unique. For, without loss of generality, assume $P$ and $D$ both the identity matrix, so that (1.1) holds. Assume $Q$ and $E$ given so that $Q A E$ satisfies (1.1). If $Q$ is not the identity permutation, then there must exist some cycle such that (say)

$$
\begin{aligned}
& a_{q_{1} q_{2}} e_{q_{2}}>a_{q_{1} q_{1}} e_{q_{1}} \\
& \cdot \cdot \cdot \\
& a_{q_{r-1} q_{r}} e_{q_{r}}>a_{q_{r-1} q_{r-1}} e_{q_{r-1}} \\
& a_{q_{r} q_{1}} e_{q_{1}}>a_{q_{r} q_{r}} e_{q_{r}} .
\end{aligned}
$$

Multiplying the inequalities (4.1) together, we obtain

$$
a_{q_{1} q_{2}} \cdots a_{q_{r} q_{1}}>a_{q_{1} q_{1}} \cdots a_{q_{r} q_{r}},
$$

which violates (1.1).

In fact, it is clear from the foregoing that the diagonal entries in the $P A D$ of (3.3) will be that collection of $n$ entries of $A$, one from each row and column, whose product is a maximum. Further, that collection is necessarily unique. Finding the collection amounts to solving the assignment problem of linear programming [1] where the "scores" are $\left\{\log a_{i j}\right\}$. In some cases this can be done easily ([4]), but not in general [6].

(ii) If we had confined our attention to real rather than complex matrices, our theorem does not apply, and the problem seems difficult. With somewhat stronger hypotheses than the real case of (3.1), the problem has been solved by Ky Fan [3].

\section{REFERENCES}

1. G. B. Dantzig, Linear Programming and Extensions, Princeton University Press, Princeton, 1963.

2. J. Desplanques, Théoréme d'Algébre, J. Math. Spec. (3) 1 (1887), 12-13.

3. Ky Fan, Note on circular disks containing the eigenvalues of a matrix, Duke Math J. 25 (1958), 441-445.

4. A. J. Hoffman, On Simple Linear Programming Problems, Proceeding, of Symposia in Pure Mathematics, Vol. III, Convexity, 1963, American Mathematical Society, pp. 317-327.

5. B. W. Levinger and R. S. Varga, Minimal Gerschgorin sets II, to be published. 6. T. S. Motzkin, The Assignment Problem, Proceedings of Symposia in Applied Mathematics, Vol. VI, Numerical Analysis, 1956, American Mathematical Society, pp. 109-125.

7. Hans Schneider, Regions of exclusion for the latent roots of a matrix, Proc. American Math. Soc. 5 (1954), 320-322.

8. Olga Taussky, A recurring theorem on determinants, Amer. Math. Monthly 56 (1949), 672-676.

9. R. S. Varga, Minimal Gerschgorin sets, to appear in Pacific J. Math.

IBM Research Center 